\title{
WHICH FINITELY GENERATED ABELIAN GROUPS ADMIT ISOMORPHIC CAYLEY GRAPHS?
}

\author{
CLARA LÖH
}

\begin{abstract}
We show that Cayley graphs of finitely generated Abelian groups are rather rigid. As a consequence we obtain that two finitely generated Abelian groups admit isomorphic Cayley graphs if and only if they have the same rank and their torsion parts have the same cardinality. The proof uses only elementary arguments and is formulated in a geometric language.
\end{abstract}

\section{INTRODUCTION}

Cayley graphs allow us to view groups as combinatorial and geometric objects. For instance, one of the main objectives of geometric group theory is to understand the relation between algebraic properties of finitely generated groups and (large scale) geometric properties of their Cayley graphs. On the other hand, the structure of Cayley graphs of finite groups plays an important role in combinatorics.

This article shows that all Cayley graphs of finitely generated Abelian groups are rather rigid (Theorem 1.3), and as a consequence that two finitely generated Abelian groups admit isomorphic Cayley graphs if and only if they have the same rank and their torsion parts have the same cardinality (Corollary 1.4).

We now describe the results in more detail. For the sake of completeness, let us briefly recall some basic notation:

Definition 1.1 (Cayley graph). Let $G$ be a group and let $S \subset G$ be a subset of $G$. The Cayley graph of $G$ with respect to $S$ is the (unlabelled, undirected) graph Cay $(G, S)$ whose vertex set is $G$ and whose set of edges is given by

$$
\left\{\{g, g \cdot s\} \mid g \in G, s \in\left(S \cup S^{-1}\right) \backslash\{e\}\right\} .
$$

Definition 1.2. Two finitely generated groups $G$ and $G^{\prime}$ admit isomorphic Cayley graphs if there exist finite generating sets $S \subset G$ and $S^{\prime} \subset G^{\prime}$ of $G$ and $G^{\prime}$ respectively such that the corresponding Cayley graphs $\operatorname{Cay}(G, S)$ and Cay $\left(G^{\prime}, S^{\prime}\right)$ are isomorphic (as unlabelled, undirected graphs).

If $G$ is a finitely generated Abelian group, then the torsion subgroup tors $G$ of $G$, i.e., the subgroup of all elements of $G$ of finite order, is a finite group. Moreover, the quotient $G$ / tors $G$ is a finitely generated free Abelian group and the rank of $G$ / tors $G$ is called the rank $\mathrm{rk}_{\mathbb{Z}} G$ of $G$.

A finitely generated group $G$ is a CI-group if the following holds [3]: Whenever $S, S^{\prime} \subset G$ are symmetric finite generating sets of $G$ such that

Date: July 23, 2012. CC C. Löh 2012, MSC 2010 classification: 05C25, 05C63, 20F65

Partially supported by the Institut Mittag-Leffler (Djursholm, Sweden). 
$\operatorname{Cay}(G, S)$ and $\operatorname{Cay}\left(G, S^{\prime}\right)$ are isomorphic, then there is a group automorphism $\varphi \in \operatorname{Aut}(G)$ with $\varphi(S)=S^{\prime}$. Based on results of Trofimov about automorphism groups of graphs, Möller and Seifter [5] showed that all finitely generated free Abelian groups (and more generally, finitely generated torsion-free nilpotent groups) are CI-groups. Later, Ryabchenko [6] provided an elementary proof of the fact that finitely generated free Abelian groups are CI-groups.

Even though not every finitely generated Abelian group is a CI-group [1, 3], we will show in the following that Cayley graphs of finitely generated Abelian groups are rigid in the following sense:

Theorem 1.3 (Cayley graph rigidity). Let $G$ and $G^{\prime}$ be finitely generated Abelian groups, and let $S \subset G$ and $S^{\prime} \subset G^{\prime}$ be finite generating sets of $G$ and $G^{\prime}$ respectively. If $\varphi$ : Cay $(G, S) \longrightarrow \operatorname{Cay}\left(G^{\prime}, S^{\prime}\right)$ is an isomorphism of graphs, then $\varphi$ induces an affine isomorphism

$$
\begin{aligned}
G / \text { tors } G & \longrightarrow G^{\prime} / \text { tors } G^{\prime} \\
{[g] } & \longmapsto[\varphi(g)]
\end{aligned}
$$

of finitely generated free Abelian groups.

The proof is based on a careful analysis of sufficiently convex geodesic lines in Cayley graphs of finitely generated Abelian groups. Similar to Ryabchenko's arguments, the key idea is that geodesic lines generated by "longest" generators satisfy a certain uniqueness property that allows to translate between the combinatorial structure of Cayley graphs and the algebraic structure of the underlying Abelian groups.

Notice however that in general not every graph automorphism of a Cayley graph of a finitely generated Abelian group is induced from an affine group automorphism (Example 3.1).

As a consequence of Theorem 1.3 we can characterise which finitely generated Abelian groups admit isomorphic Cayley graphs:

Corollary 1.4. Two finitely generated Abelian groups admit isomorphic Cayley graphs if and only if they have the same rank and their torsion parts have the same cardinality.

As a long-term perspective one might hope that a thorough understanding of the combinatorics of Cayley graphs of Abelian groups could lead to an elementary proof of quasi-isometry rigidity of virtually Abelian groups.

This article is organised as follows: In Section 2 we will study the relation between geometric and algebraic properties of geodesics in Cayley graphs of Abelian groups, which will be the main tool to prove Theorem 1.3, Section 3 contains the proof of Theorem 1.3. In Section 4 we deduce Corollary 1.4 from the theorem. Finally, for the sake of completeness Section 5 contains an alternative approach to detecting the parity of the torsion part, following a discussion on mathoverflow.net [4].

Acknowledgements. I would like to thank Lars Scheele for bringing the discussion on mathoverflow.net [4] about "Cayley graph equivalence" to my attention. Moreover, I would like to thank the Institut Mittag-Leffler for its hospitality, and Röggi Möller, Tobias Hartnick and Anders Karlsson for interesting discussions. 


\section{GEODESIC LINES IN FINITELY GENERATED AbELIAN GROUPS}

Even though Theorem 1.3 and its proof are purely combinatorial we prefer to formulate and organise the arguments in a geometric language, in terms of geodesics and suitable convexity properties. In Section 2.1 we introduce the basic geometric language for graphs and present the basic reordering argument for geodesics in Abelian groups, in Section 2.2 and Section 2.3 we study the relation between algebraic and convexity properties of geodesic lines, and in Section 2.4 we list basic properties of parallel algebraic lines in Cayley graphs.

2.1. Graphs and geodesics. In the present article, graphs are unlabelled, undirected, simple graphs. An isomorphism between graphs $(V, E)$ and $\left(V^{\prime}, E^{\prime}\right)$ is a bijective map $\varphi: V \longrightarrow V^{\prime}$ such that for all $u, v \in V$ we have that $\{u, v\} \in E$ if and only if $\{\varphi(u), \varphi(v)\} \in E^{\prime}$. If $\Gamma=(V, E)$ is a (connected) graph, then the graph structure induces a path-metric $d_{\Gamma}$ on $V$ characterised by all edges having length 1 .

For our arguments the following observation will be essential: By definition of the graph metric, any graph isomorphism is an isometry between the sets of vertices of the graphs in question. So anything that can be expressed purely in terms of metric properties of the underlying graphs will be preserved by graph isomorphisms.

Definition 2.1 (geodesic line/segment). Let $\Gamma=(V, E)$ be a graph.

- A geodesic segment in $\Gamma$ is a finite path $\left(v_{0}, \ldots, v_{n}\right)$ (of vertices) in $\Gamma$ with

$$
d_{\Gamma}\left(v_{0}, v_{n}\right)=n
$$

(equivalently, for all $j, k \in\{0, \ldots, n\}$ one has $d_{\Gamma}\left(v_{j}, v_{k}\right)=|j-k|$ ).

- A geodesic line in $\Gamma$ is an infinite path $\gamma: \mathbb{Z} \longrightarrow V$ in $\Gamma$ satisfying

$$
d_{\Gamma}(\gamma(j), \gamma(k))=|j-k|
$$

for all $j, k \in \mathbb{Z}$ (equivalently, all consecutive finite subsequences of $\gamma$ are geodesic segments).

For the sake of readability, whenever convenient we will also use the sequence notation " $\gamma_{j}:=\gamma(j)$ " for points on $\mathbb{Z}$-paths $\gamma$ in graphs.

In particular, if $G$ is a group and $S$ is a generating set of $G$, then $G$ inherits a metric $d_{S}$ from the graph structure of $\operatorname{Cay}(G, S)$; of course, this is nothing but the word metric on $G$ with respect to $S$.

One of the main points is that in Abelian groups, commutativity of the group structure allows us to generate new geodesics out of old ones by changing the order of steps (Figure 1):

Proposition 2.2 (reordering geodesic segments). Let $G$ be an Abelian group, let $S$ be a symmetric generating set of $G$, and let $\left(g_{0}, \ldots, g_{n}\right)$ be a $d_{S}$-geodesic segment in $G$. For $j \in\{0, \ldots, n-1\}$ we write

$$
s_{j}:=g_{j+1}-g_{j} \text {. }
$$

Then $s_{j} \in S$, and for every permutation $\sigma$ of $\{0, \ldots, n-1\}$ the reordered sequence

$$
\left(g_{0}, g_{0}+s_{\sigma(0)}, g_{0}+s_{\sigma(0)}+s_{\sigma(1)}, \ldots, g_{0}+s_{\sigma(0)}+\cdots+s_{\sigma(n-1)}\right)
$$

is a $d_{S^{-}}$-geodesic segment starting in $g_{0}$ and ending in $g_{n}$. 


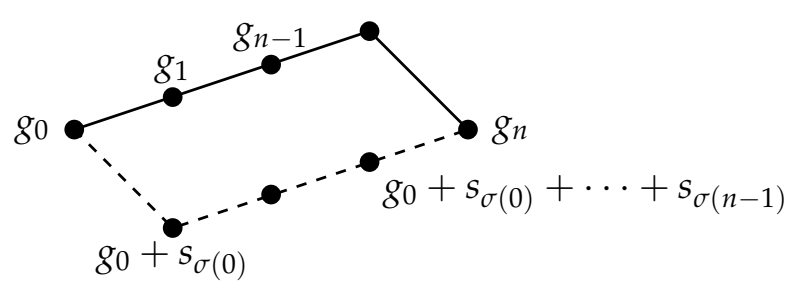

FIGURE 1. Reordering geodesic segments in Abelian groups

Proof. By definition of $\operatorname{Cay}(G, S)$, we have $s_{j} \in S$ for all $j \in\{0, \ldots, n-1\}$ and the reordered sequence is a path in $\operatorname{Cay}(G, S)$. By construction, the reordered path starts in $g_{0}$ and ends in

$$
g_{0}+\sum_{j=0}^{n-1} s_{\sigma(j)}=g_{0}+\sum_{j=0}^{n-1} s_{j}=g_{0}+\sum_{j=0}^{n-1}\left(g_{j+1}-g_{j}\right)=g_{n} .
$$

Moreover, because the reordered sequence has length $n=d_{S}\left(g_{0}, g_{n}\right)$ the claim follows.

2.2. Convex geodesic lines. As a first step, we will give the key argument linking combinatorial and algebraic structure of geodesic lines in a special and straightforward case (which, e.g., is enough for the torsion-free case).

Definition 2.3 (algebraic line). Let $G$ be an Abelian group, let $S$ be a symmetric generating set, and let $s \in S$. An algebraic line of type $s$ in $\operatorname{Cay}(G, S)$ is a $\mathbb{Z}$-path in $\operatorname{Cay}(G, S)$ of the form

$$
\begin{aligned}
& \mathbb{Z} \longrightarrow G \\
& n \longmapsto h+n \cdot s
\end{aligned}
$$

for some $h \in G$.

Definition 2.4 (convex geodesic line). Let $\Gamma=(V, E)$ be a graph. A convex geodesic line in $\Gamma$ is a geodesic line $\gamma: \mathbb{Z} \longrightarrow V$ with the following property: for all $n, m \in \mathbb{Z}$ with $n \leq m$ there is exactly one geodesic segment in $\Gamma$ starting in $\gamma_{n}$ and ending in $\gamma_{m}$, namely $\left(\gamma_{n}, \gamma_{n+1}, \ldots, \gamma_{m}\right)$.

The following proposition describes the interaction between algebraicity and convexity and shows in particular that graph isomorphisms of Cayley graphs of finitely generated free Abelian groups map algebraic lines of "maximal" type to algebraic lines.

Proposition 2.5 (convex geodesic lines vs. algebraic geodesic lines).

(1) If $G=\mathbb{Z}^{r}$ for some $r \in \mathbb{N}$, and $S \subset G$ is a (symmetric) finite generating set, and $s \in S \backslash\{0\}$ is a $\|\cdot\|_{2}$-maximal element of $S$, then all algebraic lines of type sin $\operatorname{Cay}(G, S)$ are convex geodesic lines.

(2) Graph isomorphisms map convex geodesic lines to convex geodesic lines.

(3) If $G$ is an Abelian group and $S \subset G$ is a finite subset, then every convex geodesic line in $\operatorname{Cay}(G, S)$ is algebraic. 
Proof. Ad 1: Let $h \in G$. Then

$$
\begin{aligned}
\gamma: \mathbb{Z} & \longrightarrow G \\
n & \longmapsto h+n \cdot s
\end{aligned}
$$

is a convex geodesic line in $\operatorname{Cay}(G, S)$, because: Let $n, m \in \mathbb{Z}$ with $m \geq n$. Let $k:=d_{S}\left(\gamma_{n}, \gamma_{m}\right)$ and let $\left(g_{0}, \ldots, g_{k}\right)$ be a geodesic segment in $\operatorname{Cay}(G, S)$ starting in $\gamma_{n}$ and ending in $\gamma_{m}$. Thus, $g_{j+1}-g_{j} \in S$ for all $j \in\{0, \ldots, k-1\}$ and

$$
(m-n) \cdot s=\gamma_{m}-\gamma_{n}=\sum_{j=0}^{k-1}\left(g_{j+1}-g_{j}\right) .
$$

Because $s$ is $\|\cdot\|_{2}$-maximal in $S$ and $s \neq 0$, it follows that $k \geq m-n$. Furthermore, because $\left(g_{0}, \ldots, g_{k}\right)$ is geodesic, we must have $k=m-n$ and (again by $\|\cdot\|_{2}$-maximality of $s$ ) we have $\left\|g_{j+1}-g_{j}\right\|_{2}=\|s\|_{2}$ for all $j \in$ $\{0, \ldots, k-1\}$. But now Equation 1 and elementary geometry in $\mathbb{Z}^{r} \subset \mathbb{R}^{r}$ imply that $g_{j+1}-g_{j}=s$ for all $j \in\{0, \ldots, k-1\}$. Hence,

$$
\left(g_{0}, \ldots, g_{m}\right)=(h, h+s, \ldots, h+n \cdot s)=\left(\gamma_{n}, \ldots, \gamma_{m}\right),
$$

and so $\gamma$ is a convex geodesic line in $\operatorname{Cay}(G, S)$.

Ad 2: Every graph ismorphism is an isometry with respect to the graph metrics, and isometries map convex geodesics to convex geodesics. This proves the second part.

Ad 3: Let $\gamma: \mathbb{Z} \longrightarrow G$ be a geodesic line in $\operatorname{Cay}(G, S)$ that is not algebraic. Then $\gamma$ is not a convex geodesic line: Because $\gamma$ is not algebraic, we can find $n, m \in \mathbb{Z}$ with $n<m$ and

$$
\gamma_{n+1}-\gamma_{n} \neq \gamma_{m}-\gamma_{m-1}=: s \text {. }
$$

Clearly, $s \in S$ and because $\left(\gamma_{n}, \ldots, \gamma_{m}\right)$ is a geodesic segment in $\operatorname{Cay}(G, S)$ and $G$ is Abelian, also the sequence

$$
\left(\gamma_{n}, \gamma_{n}+s, \gamma_{n+1}+s, \ldots, \gamma_{m-1}+s=\gamma_{m}\right)
$$

is a geodesic segment (Proposition 2.2). However, by construction, this sequence does not coincide with $\left(\gamma_{n}, \ldots, \gamma_{m}\right)$. So, $\gamma$ is not a convex geodesic line.

Notice that not every algebraic line whose type is of infinite order is geodesic - for example, in Cay $(\mathbb{Z},\{ \pm 1, \pm 2\})$ algebraic lines of type 1 are not geodesic.

2.3. Quasi-convex geodesic lines. In general, torsion will introduce some ambiguities in geodesics and we will not be able to find enough convex geodesic lines in Cayley graphs of finitely generated Abelian groups; for example, the graph Cay $(\mathbb{Z} \times \mathbb{Z} / 2,\{ \pm(1,0), \pm(1,1)\})$ contains no convex geodesic lines (even though the generating set consists of elements of infinite order). Therefore, we introduce the slightly weaker notions of quasialgebraic lines and quasi-convex geodesic lines.

Notation 2.6. For a finitely generated Abelian group $G$ we write

$$
\pi_{G}: G \longrightarrow G / \text { tors } G
$$

for the canonical projection. 


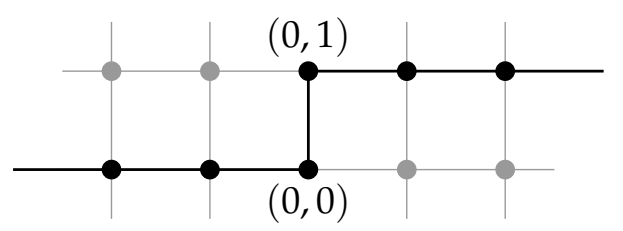

FIGURE 2. A quasi-convex geodesic line that is neither convex nor quasi-algebraic (in $\operatorname{Cay}\left(\mathbb{Z}^{2},\{ \pm(1,0), \pm(0,1)\}\right)$ )

Definition 2.7 (quasi-algebraic line). Let $G$ be a finitely generated Abelian group, let $S \subset G$ be a (symmetric) generating set, and let $s \in S$. A quasialgebraic line of quasi-type $\pi_{G}(s)$ in $\operatorname{Cay}(G, S)$ is a $\mathbb{Z}$-path $\gamma: \mathbb{Z} \longrightarrow G$ in Cay $(G, S)$ with the property that for all $n \in \mathbb{Z}$ we have

$$
\pi_{G}\left(\gamma_{n+1}-\gamma_{n}\right)=\pi_{G}(s) \in G / \text { tors } G \text {. }
$$

Clearly, the quasi-type of quasi-algebraic lines is well-defined.

Definition 2.8 (quasi-convex geodesic line). Let $\Gamma=(V, E)$ be a graph. A quasi-convex geodesic line in $\Gamma$ is a geodesic line $\gamma: \mathbb{Z} \longrightarrow V$ in $\Gamma$ such that for all $c \in \mathbb{R}_{\geq 0}$ there exists a $C \in \mathbb{R}_{\geq 0}$ with the following property: All geodesic segments in $\Gamma$ joining points that are $c$-close to $\gamma$ stay uniformly $C$-close to $\gamma$, i.e., for all $n, m \in \mathbb{Z}$ with $n \leq m$, all points $x, y \in \Gamma$ with $d_{\Gamma}\left(x, \gamma_{n}\right) \leq c$, $d_{\Gamma}\left(y, \gamma_{m}\right) \leq c$ and all geodesic segments $\widetilde{\gamma}:\left\{0, \ldots, d_{\Gamma}(x, y)\right\} \longrightarrow V$ from $x$ to $y$ we have for all $j \in\left\{0, \ldots, d_{\Gamma}(x, y)\right\}$ that

$$
d_{\Gamma}\left(\widetilde{\gamma}_{j}, \gamma_{n+j}\right) \leq C
$$

Notice that quasi-convexity in geometric group theory is usually associated with a slightly weaker property, and that quasi-convexity as defined above also includes a so-called fellow-traveller property.

In a general graph, not every geodesic line needs to be quasi-convex. Conversely, also not all quasi-convex geodesic lines are convex: We consider the integer square lattice $\operatorname{Cay}\left(\mathbb{Z}^{2},\{ \pm(1,0), \pm(0,1)\}\right)$ : It is not difficult to see that the sequence

$$
\ldots,(-2,0),(-1,0),(0,0),(0,1),(1,1),(2,1), \ldots
$$

is a quasi-convex geodesic line that is not convex (Figure 2). Moreover, this is an example of a quasi-convex geodesic line that is not quasi-algebraic.

However, we still have the following analogue of Proposition 2.5.

Proposition 2.9 (quasi-convex geodesic lines vs. quasi-algebraic lines).

(1) Let $G$ be a finitely generated Abelian group, let $S \subset G$ be a symmetric finite generating set of $G$, and let $\|\cdot\|_{2}$ be the $\ell^{2}$-norm on $G /$ tors $G$ induced by some chosen isomorphism $G /$ tors $G \cong \mathbb{Z}^{\text {rk }_{\mathbb{Z}} G}$. Let $s \in S \backslash$ tors $G$ be $a\left\|\pi_{G}(\cdot)\right\|_{2}$-maximal element of $S$. Then all quasi-algebraic lines of quasitype $\pi_{G}(s)$ in $\operatorname{Cay}(G, S)$ are quasi-convex geodesic lines.

(2) Graph isomorphisms map quasi-convex geodesic lines to quasi-convex geodesic lines.

(3) Let $G$ and $G^{\prime}$ be finitely generated Abelian groups, and suppose that there is an isomorphism $\varphi$ : Cay $(G, S) \longrightarrow \operatorname{Cay}\left(G^{\prime}, S^{\prime}\right)$ for certain symmetric 
finite generating sets $S \subset G, S^{\prime} \subset G^{\prime}$. Then $\varphi$ maps algebraic quasiconvex geodesic lines to quasi-algebraic quasi-convex geodesic lines.

Proof. Ad 1: Let $\gamma: \mathbb{Z} \longrightarrow G$ be a quasi-algebraic line of quasi-type $\pi_{G}(s)$. We argue similarly to the proof of the corresponding part of Proposition 2.5.

As a first step, we show that $\gamma$ is a geodesic line: To this end, let $n, m \in \mathbb{Z}$ with $n \leq m$, let $k:=d_{S}\left(\gamma_{n}, \gamma_{m}\right)$, and let $\left(g_{0}, \ldots, g_{k}\right)$ be a geodesic segment in $\operatorname{Cay}(G, S)$ starting in $\gamma_{n}$ and ending in $\gamma_{m}$. In particular, $g_{j+1}-g_{j} \in S$ for all $j \in\{0, \ldots, k-1\}$, and

$$
\begin{aligned}
(m-n) \cdot\left\|\pi_{G}(s)\right\|_{2} & =\left\|\pi_{G}\left(\gamma_{m}-\gamma_{n}\right)\right\|_{2}=\left\|\pi_{G}\left(\sum_{j=0}^{k-1}\left(g_{j+1}-g_{j}\right)\right)\right\|_{2} \\
& \leq \sum_{j=0}^{k-1}\left\|\pi_{G}\left(g_{j+1}-g_{j}\right)\right\|_{2} .
\end{aligned}
$$

Because $s$ is $\left\|\pi_{G}(\cdot)\right\|_{2}$-maximal in $S$, and $\left\|\pi_{G}(s)\right\|_{2} \neq 0$ it follows that

$$
d_{S}\left(\gamma_{n}, \gamma_{m}\right)=k \geq m-n \geq d_{S}\left(\gamma_{n}, \gamma_{m}\right) \text {. }
$$

Hence, $\left(\gamma_{n}, \ldots, \gamma_{m}\right)$ is a geodesic path in $\operatorname{Cay}(G, S)$, and it follows that $\gamma$ indeed is a geodesic line.

Why is $\gamma$ quasi-convex? Let $c \in \mathbb{R}_{\geq 0}$ and let $n, m \in \mathbb{Z}$ with $n \leq m$. Moreover, let $x, y \in G$ with $d_{S}\left(x, \gamma_{n}\right) \leq c$ and $d_{S}\left(y, \gamma_{m}\right) \leq c$, let $k:=$ $d_{S}(x, y)$, and let $\left(g_{0}, \ldots, g_{k}\right)$ be a geodesic segment in Cay $(G, S)$ starting in $x$ and ending in $y$. In view of the triangle inequality and the fact that $\gamma$ is geodesic, we hence obtain that

$$
m-n-2 \cdot c \leq k \leq m-n+2 \cdot c .
$$

Let $K$ be the number of steps in the geodesic segment $\left(g_{0}, \ldots, g_{k}\right)$ that are not of quasi-type $\pi_{G}(s)$. We now bound $K$ from above: We may assume that $K \neq 0$; then $S$ contains elements that are not quasi-type $\pm \pi_{G}(s)$.

We embed $G$ / tors $G$ (which we identified with $\mathbb{Z}^{\mathrm{rk}_{\mathbb{Z}} G}$ ) into $\mathbb{R}^{\mathrm{rk} G}$ and consider the standard scalar product on $\mathbb{R}^{\mathrm{rk}_{Z} G}$, which is compatible with our choice of $\ell^{2}$-norm on $G /$ tors $G$. Let $p: G \longrightarrow \mathbb{R} \cdot \pi_{G}(s) \subset \mathbb{R}^{\text {rk }_{Z} G}$ be the orthogonal projection onto the line in $\mathbb{R}^{\mathrm{rk}_{\mathbb{Z}} G}$ spanned by $\pi_{G}(s)$. Because $S$ is finite and $s$ is $\left\|\pi_{G}(\cdot)\right\|_{2}$-maximal in $S$, we have

$$
\mu:=\max \left\{\|p(t)\|_{2} \mid t \in S, \pi_{G}(t) \neq \pm \pi_{G}(s)\right\}<\left\|\pi_{G}(s)\right\|_{2} .
$$

Thus, $\left\|\pi_{G}(\cdot)\right\|_{2}$-maximality of $s$ and the triangle inequality yield

$$
\begin{aligned}
(m-n) \cdot\left\|\pi_{G}(s)\right\|_{2}-2 \cdot c \cdot\left\|\pi_{G}(s)\right\|_{2} & \leq\left\|p\left(g_{k}\right)-p\left(g_{0}\right)\right\|_{2} \\
& \leq K \cdot \mu+(k-K) \cdot\left\|\pi_{G}(s)\right\|_{2} \\
& \leq K \cdot \mu+(m-n+2 \cdot c-K) \cdot\left\|\pi_{G}(s)\right\|_{2},
\end{aligned}
$$

and so

$$
K \leq \frac{4 \cdot c \cdot\left\|\pi_{G}(s)\right\|_{2}}{\left\|\pi_{G}(s)\right\|_{2}-\mu}
$$

which is the desired upper bound depending only on $c$ and the geometry of $S$. 
Then the segment $\left(g_{0}, \ldots, g_{k}\right)$ is uniformly C-close to $\gamma$, where

$$
C:=c+\frac{8 \cdot c \cdot\left\|\pi_{G}(s)\right\|_{2}}{\left\|\pi_{G}(s)\right\|_{2}-\mu}+\operatorname{diam}_{d_{S}} \text { tors } G
$$

(which depends only on $c$ and the geometry of $(G, S)$ ): If $j \in\{0, \ldots, k\}$, then

$$
g_{j}-\gamma_{n+j} \in g_{0}-\gamma_{n}+\sum_{i=1}^{K_{j}} s_{i}-K_{j} \cdot s+\text { tors } G,
$$

where $s_{1}, \ldots, s_{K_{j}} \in S$ are the steps in the segment $\left(g_{0}, \ldots, g_{j}\right)$ that are not of quasi-type $\pi_{G}(s)$. Then $K_{j} \leq K$ and so

$$
d_{S}\left(g_{j}, \gamma_{n+j}\right) \leq c+2 \cdot K+\operatorname{diam}_{d_{S}} \text { tors } G \leq C,
$$

as desired. Hence, $\gamma$ is quasi-convex.

Ad 2: Every graph isomorphism is an isometry with respect to the graph metrics, and isometries map quasi-convex geodesic lines to quasi-convex geodesic lines. This proves the second part.

Ad 3: Let $\gamma: \mathbb{Z} \longrightarrow G$ be an algebraic quasi-convex geodesic line of type $s$ for some $s \in S$. By the second part, $\gamma^{\prime}:=\varphi \circ \gamma$ is a quasi-convex geodesic line in $\operatorname{Cay}\left(G^{\prime}, S^{\prime}\right)$. In order to show that $\gamma^{\prime}$ is quasi-algebraic, we proceed as follows:

- We will first show that all but a finite number of edges in $\gamma^{\prime}$ have the same quasi-type.

- We will then conclude that the quasi-types of all the edges of $\gamma^{\prime}$ are the same.

For the first step, assume for a contradiction that not all but a finite number of steps in $\gamma^{\prime}$ have the same type. Because $S^{\prime}$ is finite, then there exist $s^{\prime}, t^{\prime} \in S^{\prime}$ with $\pi_{G^{\prime}}\left(s^{\prime}\right) \neq \pi_{G^{\prime}}\left(t^{\prime}\right)$ and the following property: for all $k \in \mathbb{N}$ there is an $n_{k} \in \mathbb{N}$ such that the geodesic segment $\left(\gamma_{0}^{\prime}, \ldots, \gamma_{n_{k}}^{\prime}\right)$ contains at least $k$ steps of quasi-type $\pi_{G^{\prime}}\left(s^{\prime}\right)$ as well as at least $k$ steps of quasitype $\pi_{G^{\prime}}\left(t^{\prime}\right)$. Because $G^{\prime}$ is Abelian, the reordering

- start in $\gamma_{0}^{\prime}$,

- then take $k$ steps of quasi-type $\pi_{G^{\prime}}\left(s^{\prime}\right)$,

- then take $k$ steps of quasi-type $\pi_{G^{\prime}}\left(t^{\prime}\right)$,

- then take the remaining steps (ending in $\gamma_{n_{k}}^{\prime}$ )

of this geodesic segment also is a geodesic segment $\eta$ starting in $\gamma_{0}^{\prime}$ and ending in $\gamma_{n_{k}}^{\prime}$ (Proposition 2.2). Similarly, we obtain a geodesic segment $\widetilde{\eta}$ between $\gamma_{0}^{\prime}$ and $\gamma_{n_{k}}^{\prime}$ that first takes $k$ steps of quasi-type $\pi_{G^{\prime}}\left(t^{\prime}\right)$ and then $k$ steps of quasi-type $\pi_{G^{\prime}}\left(s^{\prime}\right)$ (Figure 3). But then

$$
\pi_{G^{\prime}}\left(\eta_{k}-\widetilde{\eta}_{k}\right)=k \cdot\left(\pi_{G^{\prime}}\left(s^{\prime}\right)-\pi_{G^{\prime}}\left(t^{\prime}\right)\right),
$$

and so $d_{S^{\prime}}\left(\eta_{k}, \widetilde{\eta}_{k}\right)$ can become arbitrarily large (because $\pi_{G^{\prime}}\left(s^{\prime}\right) \neq \pi_{G^{\prime}}\left(t^{\prime}\right)$ and tors $G^{\prime}$ is finite). This contradicts that $\gamma^{\prime}$ is a quasi-convex geodesic line.

For the second step, assume for a contradiction that not all steps of $\gamma^{\prime}$ have the same quasi-type. In view of the first step and translation invariance of $d_{S^{\prime}}$ we may assume that there are $s^{\prime}, t^{\prime} \in S^{\prime}$ with $\pi_{G^{\prime}}\left(s^{\prime}\right) \neq \pi_{G^{\prime}}\left(t^{\prime}\right)$ and

$$
\pi_{G^{\prime}}\left(\gamma_{n+1}^{\prime}-\gamma_{n}^{\prime}\right)=\pi_{G^{\prime}}\left(s^{\prime}\right)
$$




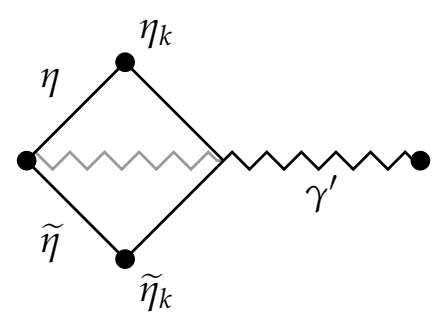

FIGURE 3. Reordered geodesics that are not close

for all $n \in \mathbb{N}_{>0}$, as well as

$$
\pi_{G^{\prime}}\left(\gamma_{1}^{\prime}-\gamma_{0}^{\prime}\right)=\pi_{G^{\prime}}\left(t^{\prime}\right)
$$

For $n, m \in \mathbb{Z}$ let $\Gamma(n, m)$ and $\Gamma^{\prime}(n, m)$ denote the number of geodesics in Cay $(G, S)$ and Cay $\left(G^{\prime}, S^{\prime}\right)$ between $\gamma_{n}$ and $\gamma_{m}$ and between $\gamma_{n}^{\prime}$ and $\gamma_{m}^{\prime}$ respectively. Because $\gamma$ is algebraic, because translations in $G$ are $d_{S}$-isometries and because $\varphi$ is an isometry with respect to the word metrics $d_{S}$ and $d_{S^{\prime}}$, we obtain

$$
\Gamma(0, m-n)=\Gamma(n, m)=\Gamma^{\prime}(n, m)
$$

for all $n, m \in \mathbb{Z}$. By definition of $\operatorname{Cay}(G, S)$, clearly

$$
\Gamma(0, k) \leq|S|^{k}
$$

for all $k \in \mathbb{N}$. Hence there is a $k \in \mathbb{N}$ with $\Gamma(0, k+1)<k \cdot \Gamma(0, k)$. On the other hand, shuffling in the step $\gamma_{1}^{\prime}-\gamma_{0}^{\prime}$ at the various positions of the geodesic segments between $\gamma_{1}^{\prime}$ and $\gamma_{k+1}^{\prime}$ produces geodesic segments (by Proposition 2.2), which are all different because $\pi_{G^{\prime}}\left(t^{\prime}\right) \neq \pi_{G^{\prime}}\left(s^{\prime}\right)$; hence

$$
(k+1) \cdot \Gamma^{\prime}(1, k+1) \leq \Gamma^{\prime}(0, k+1) .
$$

Combining these estimates, we obtain

$$
\begin{aligned}
(k+1) \cdot \Gamma(0, k) & =(k+1) \cdot \Gamma(1, k+1)=(k+1) \cdot \Gamma^{\prime}(1, k+1) \\
& \leq \Gamma^{\prime}(0, k+1)=\Gamma(0, k+1) \\
& <k \cdot \Gamma(0, k),
\end{aligned}
$$

which is a contradiction.

Therefore all steps of $\gamma^{\prime}$ have the same quasi-type, and so $\gamma^{\prime}$ is indeed a quasi-algebraic line in $\operatorname{Cay}\left(G^{\prime}, S^{\prime}\right)$.

2.4. Parallelism. We now discuss two basic properties of parallel quasialgebraic lines. On the one hand, we show that graph isomorphisms cannot map quasi-algebraic lines of the same quasi-type to quasi-algebraic lines of different quasi-types; on the other hand, we show that all quasi-algebraic lines parallel to quasi-algebraic (quasi-convex) geodesic lines must also be (quasi-convex) geodesic.

Proposition 2.10 (parallel lines and quasi-type). Let $G$ and $G^{\prime}$ be finitely generated Abelian groups, let $S \subset G$ and $S^{\prime} \subset G^{\prime}$ be symmetric finite generating sets. Let $s \in S$ and let $\gamma, \eta: \mathbb{Z} \longrightarrow G$ be quasi-algebraic lines in Cay $(G, S)$ of quasi-type $\pi_{G}(s)$. Suppose that there is a graph isomorphism $\varphi$ : Cay $(G, S) \longrightarrow$ 
$\operatorname{Cay}\left(G^{\prime}, S^{\prime}\right)$ such that $\gamma^{\prime}:=\varphi \circ \gamma$ and $\eta^{\prime}:=\varphi \circ \eta$ are quasi-algebraic lines in Cay $\left(G^{\prime}, S^{\prime}\right)$. Then $\gamma^{\prime}$ and $\eta^{\prime}$ have the same quasi-type.

Proof. Let $s^{\prime}, t^{\prime} \in S^{\prime}$ be chosen in such a way that $\pi_{G^{\prime}}\left(s^{\prime}\right)$ and $\pi_{G^{\prime}}\left(t^{\prime}\right)$ are the types of $\gamma^{\prime}$ and $\eta^{\prime}$ respectively. We now have to show that $\pi_{G^{\prime}}\left(s^{\prime}\right)=\pi_{G^{\prime}}\left(t^{\prime}\right)$, i.e, that $s^{\prime}-t^{\prime}$ lies in tors $G^{\prime}$.

In view of the definition of quasi-algebraic lines and by rearranging the torsion contributions, we find maps $h, k: \mathbb{Z} \longrightarrow$ tors $G, h^{\prime}, k^{\prime}: \mathbb{Z} \longrightarrow$ tors $G^{\prime}$ such that for all $n \in \mathbb{Z}$ we have

$$
\begin{aligned}
& \gamma_{n}=\gamma_{0}+n \cdot s+h_{n} \\
& \eta_{n}=\eta_{0}+n \cdot s+k_{n} \\
& \gamma_{n}^{\prime}=\gamma_{0}^{\prime}+n \cdot s^{\prime}+h_{n}^{\prime} \\
& \eta_{n}^{\prime}=\eta_{0}^{\prime}+n \cdot t^{\prime}+k_{n}^{\prime} .
\end{aligned}
$$

Because $\varphi$ and all translations are isometries we obtain for all $n \in \mathbb{Z}$ that

$$
\begin{aligned}
d_{S^{\prime}}\left(n \cdot\left(s^{\prime}-t^{\prime}\right),\right. & \left.\eta_{0}^{\prime}-\gamma_{0}^{\prime}+k_{n}^{\prime}-h_{n}^{\prime}\right) \\
& =d_{S^{\prime}}\left(\gamma_{0}^{\prime}+n \cdot s^{\prime}+h_{n}^{\prime}, \eta_{0}^{\prime}+n \cdot t^{\prime}+k_{n}^{\prime}\right) \\
& =d_{S^{\prime}}\left(\gamma_{n}^{\prime}, \eta_{n}^{\prime}\right) \\
& =d_{S^{\prime}}\left(\varphi\left(\gamma_{n}\right), \varphi\left(\eta_{n}\right)\right) \\
& =d_{S}\left(\gamma_{n}, \eta_{n}\right) \\
& =d_{S}\left(\gamma_{0}+n \cdot s+h_{n}, \eta_{0}+n \cdot s+k_{n}\right) \\
& =d_{S}\left(\gamma_{0}-\eta_{0}, k_{n}-h_{n}\right),
\end{aligned}
$$

and hence the triangle inequality (and translation invariance) yields

$$
d_{S^{\prime}}\left(n \cdot\left(s^{\prime}-t^{\prime}\right), \eta_{0}^{\prime}-\gamma_{0}^{\prime}\right) \leq d_{S}\left(\gamma_{0}-\eta_{0}, k_{n}-h_{n}\right)+d_{S^{\prime}}\left(k_{n}^{\prime}-h_{n}^{\prime}, 0\right) .
$$

Because tors $G$ and tors $G^{\prime}$ are finite sets, the right hand side is bounded independently of $n$. Therefore, $\left\{n \cdot\left(s^{\prime}-t^{\prime}\right) \mid n \in \mathbb{Z}\right\}$ lies in a $d_{S^{\prime}}$-ball of finite radius around $\eta_{0}^{\prime}-\gamma_{0}^{\prime}$. Moreover, as $S^{\prime}$ is finite, this ball is a finite set. Thus, $s^{\prime}-t^{\prime} \in$ tors $G^{\prime}$, as desired.

Proposition 2.11 (parallel lines stay geodesics). Let $G$ be a finitely generated Abelian group, let $S \subset G$ be a symmetric finite generating set, and let $s \in S$.

(1) If one quasi-algebraic line in $\operatorname{Cay}(G, S)$ of quasi-type $\pi_{G}(s)$ is geodesic, then all quasi-algebraic lines in $\operatorname{Cay}(G, S)$ of quasi-type $\pi_{G}(s)$ are geodesic lines.

(2) If one quasi-algebraic line in $\operatorname{Cay}(G, S)$ of quasi-type $\pi_{G}(s)$ is a quasiconvex geodesic line, then all quasi-algebraic lines in $\operatorname{Cay}(G, S)$ of quasitype $\pi_{G}(s)$ are quasi-convex geodesic lines.

Proof. Ad 1 Assume for a contradiction that there exist quasi-algebraic lines $\gamma, \eta: \mathbb{Z} \longrightarrow G$ of quasi-type $\pi_{G}(s)$ such that $\gamma$ is geodesic but $\eta$ is not. Hence, there exist $n, m \in \mathbb{Z}$ with $n \leq m$ and

$$
d_{S}\left(\eta_{n}, \eta_{m}\right) \leq m-n-1
$$


Out of $\eta$ we now construct the "periodic" quasi-algebraic line in $\operatorname{Cay}(G, S)$ of quasi-type $\pi_{G}(s)$ given by

$$
\begin{aligned}
\tilde{\eta}: \mathbb{Z} & \longrightarrow G \\
z & \longmapsto\left\lfloor\frac{z}{m-n}\right\rfloor \cdot\left(\eta_{m}-\eta_{n}\right)+\eta_{n+z \bmod (m-n)} .
\end{aligned}
$$

Because $\gamma$ and $\widetilde{\eta}$ both are of quasi-type $\pi_{G}(s)$, we have $\gamma_{k}-\widetilde{\eta}_{k} \in \gamma_{0}-\widetilde{\eta}_{0}+$ tors $G$ for all $k \in \mathbb{Z}$. Adding the facts that $d_{S}$ is translation invariant and that tors $G$ is finite we obtain

$$
d_{S}\left(\gamma_{k}, \widetilde{\eta}_{k}\right) \leq d_{S}\left(\gamma_{0}, \widetilde{\eta}_{0}\right)+\operatorname{diam}_{d_{S}} \text { tors } G
$$

for all $k \in \mathbb{Z}$. By the assumption that $\gamma$ is geodesic and the triangle inequality we therefore get

$$
\begin{aligned}
k \cdot(m-n) & =d_{S}\left(\gamma_{0}, \gamma_{k \cdot(m-n)}\right) \\
& \leq d_{S}\left(\gamma_{0}, \widetilde{\eta}_{0}\right)+d_{S}\left(\widetilde{\eta}_{0}, \widetilde{\eta}_{k \cdot(m-n)}\right)+d_{S}\left(\widetilde{\eta}_{k \cdot(m-n)}, \gamma_{k \cdot(m-n)}\right) \\
& \leq d_{S}\left(\gamma_{0}, \widetilde{\eta}_{0}\right)+d_{S}\left(0, k \cdot\left(\eta_{m}-\eta_{n}\right)\right)+d_{S}\left(\gamma_{0}, \widetilde{\eta}_{0}\right)+\operatorname{diam}_{d_{S}} \text { tors } G \\
& \leq k \cdot d_{S}\left(\eta_{n}, \eta_{m}\right)+2 \cdot d_{S}\left(\gamma_{0}, \widetilde{\eta}_{0}\right)+\operatorname{diam}_{d_{S}} \text { tors } G \\
& \leq k \cdot(m-n-1)+2 \cdot d_{S}\left(\gamma_{0}, \widetilde{\eta}_{0}\right)+\operatorname{diam}_{d_{S}} \text { tors } G
\end{aligned}
$$

for all $k \in \mathbb{Z}$, which leads to a contradiction for large enough $k$. So if $\gamma$ is geodesic, then also $\eta$ must be geodesic.

Ad 2. Any two quasi-algebraic lines of the same quasi-type stay uniformly close (because the metric $d_{S}$ is translation invariant and tors $G$ has finite diameter). By definition, any geodesic line uniformly close to a quasiconvex geodesic is itself quasi-convex. Therefore, the second part is a consequence of the first part.

\section{PRoOf of CAYLEY GRAPH RIGIDITY}

Using the properties of quasi-algebraic and quasi-convex geodesic lines established in the previous section, we now prove Theorem 1.3 .

Proof (of Theorem 1.3). Of course, we may assume that $S$ and $S^{\prime}$ are symmetric; moreover, because translations induce isomorphisms on the corresponding Cayley graphs, we can assume that $\varphi(0)=0$. We prove the theorem by induction on $S$.

For the base case, we suppose that $S$ consists of torsion elements (in particular, this contains the case $S=\varnothing$ and $G=\{0\}$ ). In this case, $G=$ tors $G$, and the theorem clearly holds.

For the induction step we now may assume that $S$ contains at least one non-torsion element and that the theorem holds for all subgroups generated by proper subsets of $S$. We choose a basis of $G /$ tors $G \cong \mathbb{Z}^{\text {rk }_{\mathbb{Z}} G}$ and consider the corresponding $\ell^{2}$-norm on $G /$ tors $G$. Let $s \in S$ be a $\left\|\pi_{G}(\cdot)\right\|_{2^{-}}$ maximal element of $S$; because $S$ contains non-torsion elements, $s \notin$ tors $G$. Let us fix some notation: We write $s^{\prime}:=\varphi(s)$ and

$$
\begin{aligned}
S(s) & :=\left\{t \in S \mid \pi_{G}(t)=\pi_{G}(s)\right\}, \\
S^{\prime}\left(s^{\prime}\right) & :=\left\{t^{\prime} \in S^{\prime} \mid \pi_{G}\left(t^{\prime}\right)=\pi_{G}\left(s^{\prime}\right)\right\},
\end{aligned}
$$


as well as

$$
S_{s}:=S \backslash(S(s) \cup-S(s)), \quad S_{s^{\prime}}^{\prime}:=S^{\prime} \backslash\left(S^{\prime}\left(s^{\prime}\right) \cup-S^{\prime}\left(s^{\prime}\right)\right) .
$$

We then let $G_{S}$ and $G_{S}^{\prime}$ be the subgroups of $G$ and $G^{\prime}$ generated by $S_{S}$ and $S_{s^{\prime}}^{\prime}$ respectively.

We will now proceed in the following steps:

(1) We show that $\varphi$ turns algebraic lines of type in $S(s)$ into quasialgebraic lines of quasi-type $\pi_{G^{\prime}}\left(s^{\prime}\right)$; in particular, we obtain for all $y \in G$, all $t \in S(s)$ and all $m \in \mathbb{Z}$ that

$$
\pi_{G^{\prime}} \circ \varphi(y+m \cdot t)=\pi_{G^{\prime}} \circ \varphi(y)+m \cdot \pi_{G^{\prime}}\left(s^{\prime}\right) .
$$

(2) We show that $\varphi$ induces an isomorphism $\varphi_{s}$ between Cay $\left(G_{S}, S_{S}\right)$ and $\operatorname{Cay}\left(G_{s^{\prime}}^{\prime}, S_{s^{\prime}}^{\prime}\right)$.

(3) We apply the induction hypothesis to $\varphi_{s}$.

(4) Using the knowledge about $\varphi_{s}$ and algebraic lines of type in $S(s)$ we show that

$$
\pi_{G^{\prime}} \circ \varphi: G \longrightarrow G^{\prime} / \text { tors } G^{\prime}
$$

is additive.

(5) We conclude that the map

$$
\begin{aligned}
\bar{\varphi}: G / \text { tors } G & \longrightarrow G^{\prime} / \text { tors } G^{\prime} \\
{[g] } & \longmapsto[\varphi(g)]
\end{aligned}
$$

is indeed well-defined and that $\bar{\varphi}$ is an affine isomorphism.

Ad 1](quasi-algebraic lines): Because $s$ is $\left\|\pi_{G}(\cdot)\right\|_{2}$-maximal and $s \notin$ tors $G$, quasi-algebraic lines of quasi-type $\pi_{G}(s)$ in $G$ are quasi-convex geodesic lines (Proposition 2.9(1)). As $\varphi$ is a graph isomorphism, $\varphi$ therefore maps algebraic lines of any type in $S(s)$ to quasi-algebraic quasi-convex geodesic lines (Proposition 2.9 (3)), which all are of the same quasi-type (Proposition 2.10). Looking at the algebraic line of type $s$ through 0 shows that they all have quasi-type $\pi_{G^{\prime}}\left(s^{\prime}\right)$.

Ad2 (reduced Cayley graphs): We first show that $\varphi$ yields a graph isomorphism $\operatorname{Cay}\left(G, S_{s}\right) \longrightarrow \operatorname{Cay}\left(G^{\prime}, S_{s^{\prime}}\right)$ : In view of the first part it suffices to show that all algebraic lines in $G^{\prime}$ of type in $S^{\prime}\left(s^{\prime}\right)$ are indeed $\varphi$-images of quasi-algebraic lines in $G$ of quasi-type $\pi_{G}(s)$. By the first step, at least one quasi-algebraic line in $G^{\prime}$ of quasi-type $\pi_{G^{\prime}}\left(s^{\prime}\right)$ is a quasi-convex geodesic line. Thus, by parallelism (Proposition 2.11), all quasi-algebraic lines of quasi-type $\pi_{G^{\prime}}\left(s^{\prime}\right)$ in $G^{\prime}$ are quasi-convex geodesic lines.

Let $\psi: \operatorname{Cay}\left(G^{\prime}, S^{\prime}\right) \longrightarrow \operatorname{Cay}(G, S)$ be the graph isomorphism inverse to $\varphi$. The previous paragraph shows that we can apply Proposition 2.9 (3) to deduce that $\psi$ maps algebraic lines of type in $S^{\prime}\left(s^{\prime}\right)$ to quasi-algebraic lines in $G$. Again, parallelism shows that these quasi-algebraic lines in $G$ must all be of quasi-type $\pi_{G}(s)$ (Proposition 2.10).

Therefore, $\varphi$ induces a graph isomorphism Cay $\left(G, S_{s}\right) \longrightarrow \operatorname{Cay}\left(G^{\prime}, S_{S^{\prime}}^{\prime}\right)$. This graph isomorphism maps connected components to connected components; the connected component of 0 in $\operatorname{Cay}\left(G, S_{s}\right)$ is $\operatorname{Cay}\left(G_{S}, S_{s}\right)$, and the connected component of $\varphi(0)=0$ in $\operatorname{Cay}\left(G^{\prime}, S_{s^{\prime}}^{\prime}\right)$ is Cay $\left(G_{s^{\prime}}^{\prime}, S_{s^{\prime}}^{\prime}\right)$. Hence, $\varphi$ induces a graph isomorphism $\operatorname{Cay}\left(G_{s}, S_{s}\right) \longrightarrow \operatorname{Cay}\left(G_{s^{\prime}}^{\prime}, S_{s^{\prime}}^{\prime}\right)$. 
Ad 3 (induction): By construction, $G_{s}, G_{s^{\prime}}^{\prime}$ are finitely generated Abelian groups, generated by $S_{s}$ and $S_{S^{\prime}}^{\prime}$ respectively. By induction, the map

$$
\begin{aligned}
\bar{\varphi}_{s}: G_{s} / \text { tors } G_{s} & \longrightarrow G_{s^{\prime}}^{\prime} / \text { tors } G_{s^{\prime}}^{\prime} \\
{[g] } & \longmapsto\left[\varphi_{s}(g)\right]
\end{aligned}
$$

is well-defined and an affine isomorphism. Because of $\varphi(0)=0$, it follows that $\bar{\varphi}_{s}(0)=0$ and so $\bar{\varphi}_{s}$ is a group isomorphism. In particular, also the map $\pi_{G_{s^{\prime}}^{\prime}} \circ \varphi_{s}=\bar{\varphi}_{s} \circ \pi_{G_{s}}$ is a group homomorphism; hence, also $\pi_{G^{\prime}} \circ \varphi_{s}$ is additive.

Ad 4 (additivity): Let $x, \widetilde{x} \in G$. We can write $x$ and $\widetilde{x}$ in the form

$$
x=y+\sum_{j=1}^{k} m_{j} \cdot s_{j}, \quad \widetilde{x}=\widetilde{y}+\sum_{j=1}^{\widetilde{k}} \widetilde{m}_{j} \cdot \widetilde{s}_{j},
$$

where $y, \widetilde{y} \in G_{s}$, and $k, \widetilde{k} \in \mathbb{N}, m_{1}, \ldots, m_{k}, \widetilde{m}_{1}, \ldots, \widetilde{m}_{\widetilde{k}} \in \mathbb{Z}$, as well as $s_{1}, \ldots, s_{k}, \widetilde{s}_{1}, \ldots, \widetilde{s_{\tilde{k}}} \in S(s)$. In view of the first step we obtain

$$
\begin{aligned}
\pi_{G^{\prime}} \circ \varphi(x) & =\pi_{G^{\prime}} \circ \varphi(y)+\sum_{j=1}^{k} m_{j} \cdot \pi_{G^{\prime}}\left(s^{\prime}\right), \\
\pi_{G^{\prime}} \circ \varphi(\widetilde{x}) & =\pi_{G^{\prime}} \circ \varphi(\widetilde{y})+\sum_{j=1}^{\widetilde{k}} \widetilde{m}_{j} \cdot \pi_{G^{\prime}}\left(s^{\prime}\right), \\
\pi_{G^{\prime}} \circ \varphi(x+\widetilde{x}) & =\pi_{G^{\prime}} \circ \varphi(y+\widetilde{y})+\sum_{j=1}^{k} m_{j} \cdot \pi_{G^{\prime}}\left(s^{\prime}\right)+\sum_{j=1}^{\widetilde{k}} \widetilde{m}_{j} \cdot \pi_{G^{\prime}}\left(s^{\prime}\right) .
\end{aligned}
$$

Because $y, \widetilde{y} \in G_{s}$ and because $\pi_{G^{\prime}} \circ \varphi_{s}$ is additive by the third step, we conclude that

$$
\begin{aligned}
\pi_{G^{\prime}} \circ \varphi(x+\widetilde{x}) & =\pi_{G^{\prime}} \circ \varphi(y)+\pi_{G^{\prime}} \circ \varphi(\widetilde{y})+\sum_{j=1}^{k} m_{j} \cdot \pi_{G^{\prime}}\left(s^{\prime}\right)+\sum_{j=1}^{\widetilde{k}} \widetilde{m}_{j} \cdot \pi_{G^{\prime}}\left(s^{\prime}\right) \\
& =\pi_{G^{\prime}} \circ \varphi(x)+\pi_{G^{\prime}} \circ \varphi(\widetilde{x}),
\end{aligned}
$$

as desired.

Ad[5](affine isomorphism): By the previous step, $\pi_{G^{\prime}} \circ \varphi: G \longrightarrow G^{\prime} /$ tors $G^{\prime}$ is additive. Because $\varphi(0)=0$ it follows that $\pi_{G^{\prime}} \circ \varphi$ is a group homomorphism. Hence, $\pi_{G^{\prime}} \circ \varphi$ maps tors $G$ to tors $\left(G^{\prime} /\right.$ tors $\left.G^{\prime}\right)=\{0\}$. Therefore, $\bar{\varphi}$ is well-defined, and a group homomorphism.

Applying the same arguments to the graph isomorphism inverse to $\varphi$ we see that the affine homomorphism $\bar{\varphi}$ has an affine inverse, and so is an affine isomorphism.

This completes the proof of Theorem 1.3 .

The following example shows that in the theorem it is essential that we divide out the torsion part: In general, not every isomorphism between Cayley graphs of finitely generated Abelian groups is induced from an affine isomorphism between the groups: 

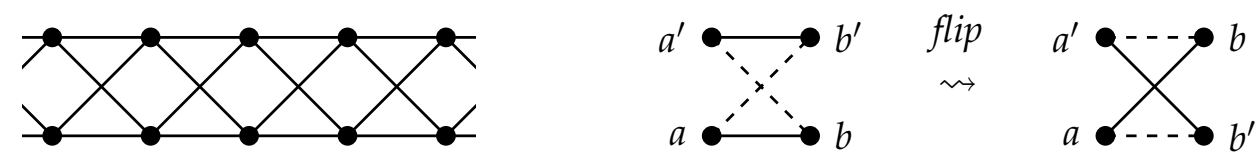

FIGURE 4 . The graph $\operatorname{Cay}(\mathbb{Z} \times \mathbb{Z} / 2,\{ \pm(1,0), \pm(1,1)\})$ and the effect of flipping

(Non-)Example 3.1. We consider the graph Cay $(\mathbb{Z} \times \mathbb{Z} / 2,\{ \pm(1,0), \pm(1,1)\})$ : For any $n \in \mathbb{Z}$ the flip at position $n$, i.e., the map

$$
\begin{aligned}
\mathbb{Z} \times \mathbb{Z} / 2 & \longmapsto \mathbb{Z} \times \mathbb{Z} / 2 \\
(x, y) & \longmapsto \begin{cases}(x, y) & \text { if } x \neq n \\
(x, 1-y) & \text { if } x=n,\end{cases}
\end{aligned}
$$

is a graph automorphism of $\operatorname{Cay}(\mathbb{Z} \times \mathbb{Z} / 2,\{ \pm(1,0), \pm(1,1)\})$ (Figure 4), but this graph automorphism is not induced by an affine isomorphism of $\mathbb{Z} \times \mathbb{Z} / 2$ (even though $\mathbb{Z}$ and $\mathbb{Z} / 2$ are CI-groups).

\section{EXTRACTING THE SIZE OF THE TORSION PART}

We will now deduce Corollary 1.4 from Theorem 1.3 .

Proof (of Corollary 1.4). Let $G$ and $G^{\prime}$ be finitely generated Abelian groups with $\mathrm{rk}_{\mathbb{Z}} G=\mathrm{rk}_{\mathbb{Z}} G^{\prime}$ and $\mid$ tors $G|=|$ tors $G^{\prime} \mid$. We write

$$
d:=\operatorname{rk}_{\mathbb{Z}} G=\operatorname{rk}_{\mathbb{Z}} G^{\prime} \text { and } k:=\mid \text { tors } G|=| \text { tors } G^{\prime} \mid .
$$

Let $S$ and $S^{\prime}$ be $\mathbb{Z}$-bases of $G /$ tors $G \cong \mathbb{Z}^{d}$ and $G^{\prime} /$ tors $G^{\prime} \cong \mathbb{Z}^{d}$ respectively. Because of $G \cong \mathbb{Z}^{d} \times$ tors $G$ and $G^{\prime} \cong \mathbb{Z}^{d} \times$ tors $G^{\prime}$ we have

$$
\operatorname{Cay}(G, S \cup \text { tors } G) \cong \operatorname{Cay}\left(\mathbb{Z}^{d},\left\{e_{1}, \ldots, e_{d}\right\}\right) \square K_{k} \cong \operatorname{Cay}\left(G^{\prime}, S^{\prime} \cup \text { tors } G^{\prime}\right),
$$

where $\left(e_{1}, \ldots, e_{d}\right)$ is the standard basis of $\mathbb{Z}^{d}$, where $K_{k}$ is the complete graph on $k$ vertices, and " $\square$ " denotes the Cartesian product of graphs. So $G$ and $G^{\prime}$ admit isomorphic Cayley graphs.

Conversely, let $G$ and $G^{\prime}$ be finitely generated Abelian groups that admit isomorphic Cayley graphs, i.e., there are finite generating sets $S \subset G$, $S^{\prime} \subset G^{\prime}$ such that there is an isomorphism $\varphi: \operatorname{Cay}(G, S) \longrightarrow \operatorname{Cay}\left(G^{\prime}, S^{\prime}\right)$. By Theorem 1.3, the graph isomorphism $\varphi$ induces an affine isomorphism $\mathbb{Z}^{\mathrm{rk}_{\mathbb{Z}} G} \cong G /$ tors $G \longrightarrow G^{\prime} /$ tors $G^{\prime} \cong \mathbb{Z}^{\mathrm{rk}_{\mathbb{Z}} G^{\prime}}$.

Because affine isomorphisms between finitely generated Abelian free groups are rank-preserving we obtain $\mathrm{rk}_{\mathbb{Z}} G=\mathrm{rk}_{\mathbb{Z}} G^{\prime}$.

Moreover, the fact that $\varphi$ induces a well-defined map between the quotients shows that $\varphi$ (tors $G) \subset \varphi(0)+$ tors $G^{\prime}$. Because $\varphi$ is bijective it follows that

$$
\mid \text { tors } G|\leq| \text { tors } G^{\prime} \mid \text {. }
$$

Applying the same argument to $\varphi^{-1}$ shows the reverse inequality, and hence we obtain $\mid$ tors $G|=|$ tors $G^{\prime} \mid$. 
Of course, we cannot recover the complete algebraic structure of the torsion part - there are finite Abelian groups of the same cardinality that are not isomorphic.

Another way to see that finitely generated Abelian groups that admit isomorphic Cayley graphs have the same rank uses the growth rate: The rank of a finitely generated Abelian group coincides with the growth rate of the group [2, Chapter VI] and the growth rate is preserved by graph isomorphisms.

Problem 4.1. Can also the size of the torsion part of a finitely generated Abelian group be extracted from the growth function of every Cayley graph (with respect to a finite generating set)?

In Section 5, we will see that at least the parity of the torsion part can be detected in this way. However, the general case seems to be open.

\section{DETECTING THE PARITY OF THE TORSION PART, ALTERNATIVE APPROACH}

The discussion on mathoverflow.net [4] about whether the relation "admit isomorphic Cayley graphs" is transitive for finitely generated groups contains a neat argument by G. Hainke and L. Scheele that allows to distinguish Cayley graphs of $\mathbb{Z}$ from Cayley graphs of $\mathbb{Z} \times \mathbb{Z} / 2$. The idea is that for Abelian groups taking inverses leads to automorphisms of Cayley graphs and that counting fixed points of these automorphisms reveals the $\mathbb{Z} / 2$-factor.

In the following, we show how the same argument can be used to reveal the parity of the torsion part of finitely generated Abelian groups:

Proposition 5.1 (parity of the torsion part via size of balls). Let $G$ be a finitely generated Abelian group and let $S \subset G$ be a finite generating set. Moreover, let $r \in \mathbb{N}$ with $r>\operatorname{diam}_{d_{S}}$ tors $G$. Then

$$
\beta(r) \equiv \mid \text { tors } G \mid \bmod 2,
$$

where $\beta(r)$ is the number of elements of the $d_{S}$-ball $B_{S}(r)$ of radius $r$ around 0 in $G$.

Proof. Because $G$ is Abelian, the map

$$
\begin{aligned}
\tau: G & \longrightarrow G \\
g & \longmapsto-g
\end{aligned}
$$

is an automorphism of $\operatorname{Cay}(G, S)$. Because $\tau(0)=0$ and because graph isomorphisms are isometries with respect to the graph metrics it follows that $\tau\left(B_{S}(r)\right)=B_{S}(r)$. Moreover, $\tau \circ \tau=\mathrm{id}_{G}$, and the fixed points of $\tau$ are exactly the elements of $G$ of order at most 2; in particular, all fixed points of $\tau$ lie in tors $G$ and hence in $B_{S}(r)$. Therefore,

$$
\beta(r)=\left|B_{S}(r)\right| \equiv|\{g \in G \mid \tau(g)=g\}| \bmod 2,
$$

and thus

$$
\beta(r) \equiv \mid\{g \in G \mid g \text { has order at most } 2\} \mid \bmod 2 .
$$

Now counting the number of elements of order 2 in $G$ finishes the proof (see Lemma5.2 below). 
Lemma 5.2 (number of elements of order at most 2). Let $G$ be a finite Abelian group. Then

$$
\mid\{g \in G \mid g \text { has order at most } 2\}|\equiv| G \mid \bmod 2 .
$$

Proof. Because $G$ is a finite Abelian group, we can write

$$
G \cong A \times \prod_{i \in I} B_{i}
$$

where $A$ is a finite Abelian group of odd order, $I$ is a finite set, and all $B_{i}$ are finite cyclic groups of even order. An element of $G$ has order at most 2 if and only if all of its components in the above product decomposition have order at most 2 . The group $A$ has exactly one element of order at most 2 (namely, 0 ), and each of the $B_{i}$ has exactly two such elements (namely, 0 and the element corresponding to $\left.\left|B_{i}\right| / 2\right)$. Hence, $G$ has exactly $2^{|I|}$ elements of order at most 2. Because $|G| \equiv 2^{|I|} \bmod 2$, the claim follows.

\section{REFERENCES}

[1] B. Elspas, J. Turner. Graphs with circulant adjacency matrices. J. Combinatorial Theory 9, pp. 297-307, 1970. Cited on page:2

[2] P. de la Harpe. Topics in Geometric Group Theory. University of Chicago Press, 2000. Cited on page: 15

[3] C.H. Li. On isomorphisms of finite Cayley graphs - a survey. Discrete Math. 256, no. 12, pp. 301-334, 2002. Cited on page:112

[4] Cayley graphs of finitely generated groups. Discussion on mathoverflow, http://mathoverflow.net/questions/18766/cayley-graphs-of-finitely-generated-groups, last accessed on February 23, 2012. Cited on page:2,15

[5] R. Möller, N. Seifter. Digraphical regular representations of infinite finitely generated groups. European J. Combin. 19, no. 5, pp. 597-602, 1998. Cited on page:2

[6] A.A. Ryabchenko. Isomorphisms of Cayley graphs of a free abelian group. (Russian. Russian summary) Sibirsk. Mat. Zh. 48, no. 5, pp. 1142-1146, 2007.

translation in Siberian Math. J. 48, no. 5, pp. 919-922, 2007. Cited on page:2

Clara Löh

Fakultät für Mathematik

Universität Regensburg

93040 Regensburg

Germany

clara.loeh@mathematik.uni-regensburg.de

http://www.mathematik.uni-regensburg.de/loeh 